\title{
Detection of Early Invasion on the Basis of Basement Membrane Destruction in Small Adenocarcinomas of the Lung and Its Clinical Implications
}

\author{
Koichi Goto, M.D., Tomoyuki Yokose, M.D., Tetsuro Kodama, M.D., Kanji Nagai, M.D., \\ Yutaka Nishiwaki, M.D., Masayuki Ando, M.D., Kiyoshi Mukai, M.D., Atsushi Ochiai, M.D. \\ Pathology Division, National Cancer Center Research Institute East (KG, TY, KM, AO), Kashiwa, Chiba; \\ Division of Thoracic Oncology, National Cancer Center Hospital East (KG, KN, YN), Kashiwa, Chiba; \\ Division of Medical Oncology, National Cancer Center Hospital (TK), Tsukiji, Tokyo; and First Department \\ of Internal Medicine, Kumamoto University School of Medicine (KG, MA), Kumamoto, Japan
}

To evaluate the correlation between the degree of basement membrane $(\mathrm{BM})$ preservation and clinicopathological characteristics in the replacementgrowth type (lepidic growth type) of small peripheral adenocarcinomas of the lung, the $B M$ components of 72 surgically resected replacementgrowth type adenocarcinomas of the lung, $2 \mathrm{~cm}$ or less in diameter, were evaluated immunohistochemically by using a monoclonal antibody to Type IV collagen and polyclonal antibodies to $7 \mathrm{~S}$ collagen and laminin. The tumors were classified into the following three distinctive histological types according to the condition of the elastic framework: Type I, bronchioloalveolar carcinoma without fibrotic foci; Type II, sclerosing bronchioloalveolar carcinoma without elastic framework destruction; and Type III, sclerosing bronchioloalveolar carcinoma with elastic framework destruction. The BM was well preserved in the area of bronchioloalveolar spread along fully expanded alveoli in all tumor types; however, BM preservation was significantly lost in the areas of collapsed alveoli in Type III tumors. There were no $\mathrm{BM}$ component staining reactions in the scarred regions of Type III tumors. In addition, lymph node metastasis was significantly greater in Type III tumors and BM-destroyed tumors. We concluded that the BM was largely destroyed by tumor cell invasion in the scarred region of Type III adenocarcinomas. Type III tumors had discontinuous

Copyright $(92001$ by The United States and Canadian Academy of Pathology, Inc.

VOL. 14, NO. 12, P. 1237, 2001 Printed in the U.S.A.

Date of acceptance: August 13, 2001.

This work was supported in part by Grants-in-Aid for the Second Term Comprehensive 10-Year Strategy for Cancer Control from the Ministry of Health and Welfare of Japan.

Address reprint requests to: Atsushi Ochiai, M.D., Pathology Division, National Cancer Center Research Institute East, 6-5-1 Kashiwanoha, Kashiwa, Chiba 277-8577, Japan; e-mail: aochiai@east.ncc.go.jp; fax: 81-471-34-6865.

BMs in the area of collapsed alveoli, indicating that this BM-destructive pattern must be the first step in tumor invasion. Type I and II tumors were concluded to be noninvasive adenocarcinomas, because their BM components were well preserved and they had a good outcome.

KEY WORDS: Adenocarcinoma, Basement membrane, Bronchioloalveolar carcinoma, Early invasion, Laminin, Lung cancer, Type IV collagen.

Mod Pathol 2001;14(12):1237-1245

Surgical resection is the standard therapy for clinical stage I non-small cell lung cancer (NSCLC). However, the 5-year survival rate in this stage has been reported to be $60-80 \%$ even after curative resection (1-5). Although Stage I carcinomas are thought to be early carcinomas curable by surgery alone, pathological examination sometimes reveals lymph node or intrapulmonary metastasis. Therefore, the preoperative clinical stage must often be underestimated, and some Stage I tumors are already advanced and have a poor prognosis.

Shimosato et al. (6) reported the prognostic implications of fibrotic foci in small peripheral lung cancer. They found that adenocarcinomas with fibrotic foci consisting of collagenization or hyalinization had a greater incidence of lymph node metastasis and blood vessel invasion. Consequently, these tumors had a poorer outcome than those without fibrotic foci. In addition, Noguchi et al. (7) reported that small localized bronchioloalveolar carcinomas (LBACs), less than $2 \mathrm{~cm}$ in diameter, with or without structural collapse of the alveoli, had no lymph node metastasis and an excellent prognosis (100\% 5-year survival rate); however, small LBACs with active fibroblastic proliferation had a poorer outcome ( $75 \%$ 5-year survival rate). The latter category tends to behave the same as 
LBAC in the advanced stages. These results suggest that early invasion begins in small adenocarcinomas with fibrotic foci (8).

Liotta and colleagues (9-11) have proposed a model of invasion and metastasis by cancer cells in which the basement membrane (BM) plays an important role as a barrier against tumor cell invasion (12). There have been several reports claiming that disruption of the BM in various tumors significantly correlated with a high incidence of distant metastasis and therefore with reduced patient survival (13-16). In another report, however, discontinuity of the BM was found to be of little prognostic significance (17). The significance of BM destruction in various studies may differ because different methods of BM evaluation were used.

In this report, the degree of $\mathrm{BM}$ preservation was evaluated by immunohistochemical staining for laminin, Type IV, and 7S collagen, all of which are major components of the BM (18). Three different areas of the same tumor identified on the basis of having the structural characteristics of the replacement-growth type (lepidic growth type) of adenocarcinoma were studied, and the immunohistochemical results were correlated with the histological type, lymph node metastasis, and survival.

\section{MATERIALS AND METHODS}

\section{Tissues Specimens}

We histologically reviewed 123 peripheral adenocarcinomas of the lung having a maximum tumor diameter of $2 \mathrm{~cm}$ or less. The specimens were surgically resected between 1983 and 1995 at the National Matsudo Hospital and National Cancer Center Hospital East. None of the cases had been treated by radiotherapy or chemotherapy preoperatively. Two pathologists (T.Y., T.K.) reviewed hematoxylin and eosin (HE) -stained slides of these tumors, and only the 72 tumors possessing replacement growth were selected for this study. The replacement-growth type of adenocarcinoma shows growth of well-differentiated cuboidal or columnar tumor cells replacing alveolar epithelium, as a single layer, with minimal or mild thickening of the alveolar septa (7). When the World Health Organization (WHO) pathological criteria for adenocarcinoma of the lung was applied to our series, all our tumors were included in bronchioloalveolar carcinoma or adenocarcinoma mixed bronchioloalveolar subtype (19). The clinical characteristics of the 72 patients are given in Table 1. Their median age was 61 years, and 54 patients were female. Fifty-nine patients, consisting of 57 with Stage IA disease and 2 with Stage IB disease, were negative for lymph node
TABLE 1. Patient Characteristics

\begin{tabular}{ll}
\hline \multicolumn{1}{c}{ Characteristic } & Data \\
\hline Patients, $n$ & 72 \\
Age in y, median (range) & $61(28-85)$ \\
Male/female $(n)$ & $18 / 54$ \\
Pathologic stage $(n)$ & \\
IA & 57 \\
IB & 2 \\
IIA & 6 \\
IIIA & 4 \\
IIIB & 3 \\
Histological type $(n)$ & \\
I & 2 \\
II & 17 \\
III & 53 \\
\hline
\end{tabular}

metastasis. Lymph node or intrapulmonary metastases were pathologically detected in the remaining 13 patients.

\section{Immunohistochemistry}

Paraffin blocks of formalin-fixed tissue were serially sectioned at $4 \mu \mathrm{m}$. One section was stained with HE for histological evaluation, another section was stained with Victoria blue to demonstrate the bronchioloalveolar structure, and the remaining sections were used for immunohistochemical staining for Type IV collagen, 7S collagen, and laminin. The primary antibodies used were a monoclonal antibody to Type IV collagen (Fuji Chemical Industries, Toyama, Japan; 1:100), a polyclonal antibody to 7S collagen (Nippon DPC Co., Tokyo, Japan; 1:1000), and two polyclonal antibodies to laminin (EY Laboratories, San Mateo, CA, 1:50 and DAKO, Glostrup, Denmark, 1:500). We mixed two polyclonal antibodies against laminin to intensify the immunoreactivity. The sections were treated with protease (Type XXIV, Sigma, St. Louis, MO) to enhance immunoreactivity. An ABC kit (DAKO) was used to visualize antigen localization with diaminobenzidine as a chromogen.

\section{Evaluation}

The degree of BM preservation at the tumorstromal interface was scored as complete preservation (CP); partial destruction (PD), which indicated that even a part of the BM was destroyed; or complete destruction (CD; Fig. 1). We evaluated BM preservation in the following three areas within the same tumor (Fig. 2): an area with a well-preserved lepidic growth (LG) pattern; an area of collapsed alveoli, and a scarred area. The LG region was present at the periphery of the tumor, which abutted normal lung, where the tumor cells showed a replacement growth pattern on the walls of the preexisting alveoli. The area of collapsed alveoli showed formation of fibrotic foci caused by col- 


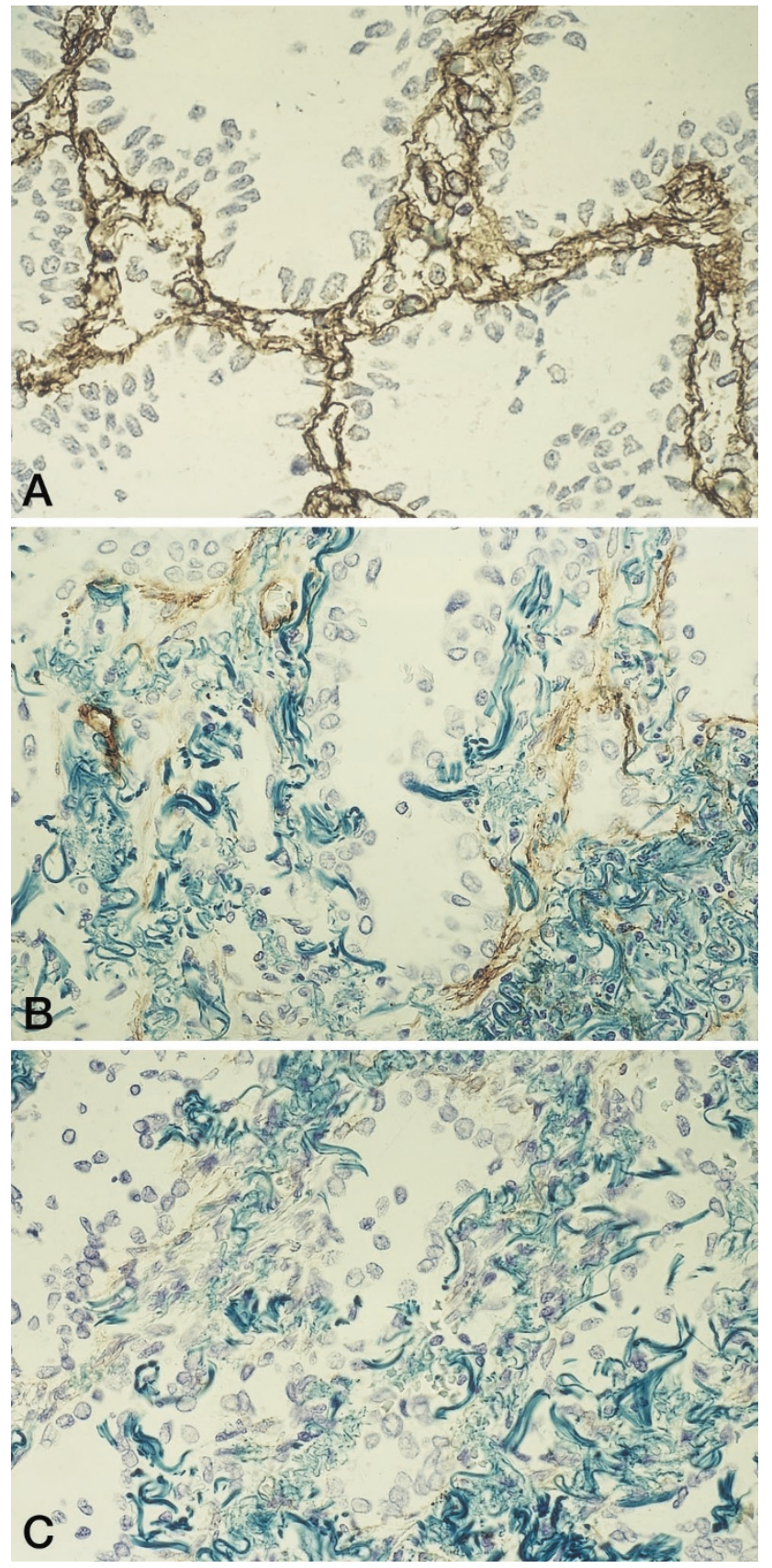

FIGURE 1. Grades of basement membrane destruction (Type IV collagen). A, complete preservation (CP). B, partial destruction (PD). C, complete destruction (CD).

lapse of the alveoli, where elastic fibers were markedly increased and the alveolar framework was collapsed but not destroyed. The scarred region was the fibrotic focus in the central zone of the tumor, with an abundant amount of collagen fibers where the elastic fibers had obviously been destroyed.

Lymph vessel invasion and vascular invasion was evaluated anywhere and just outside the tumor on the HE- and Victoria blue-stained sections.
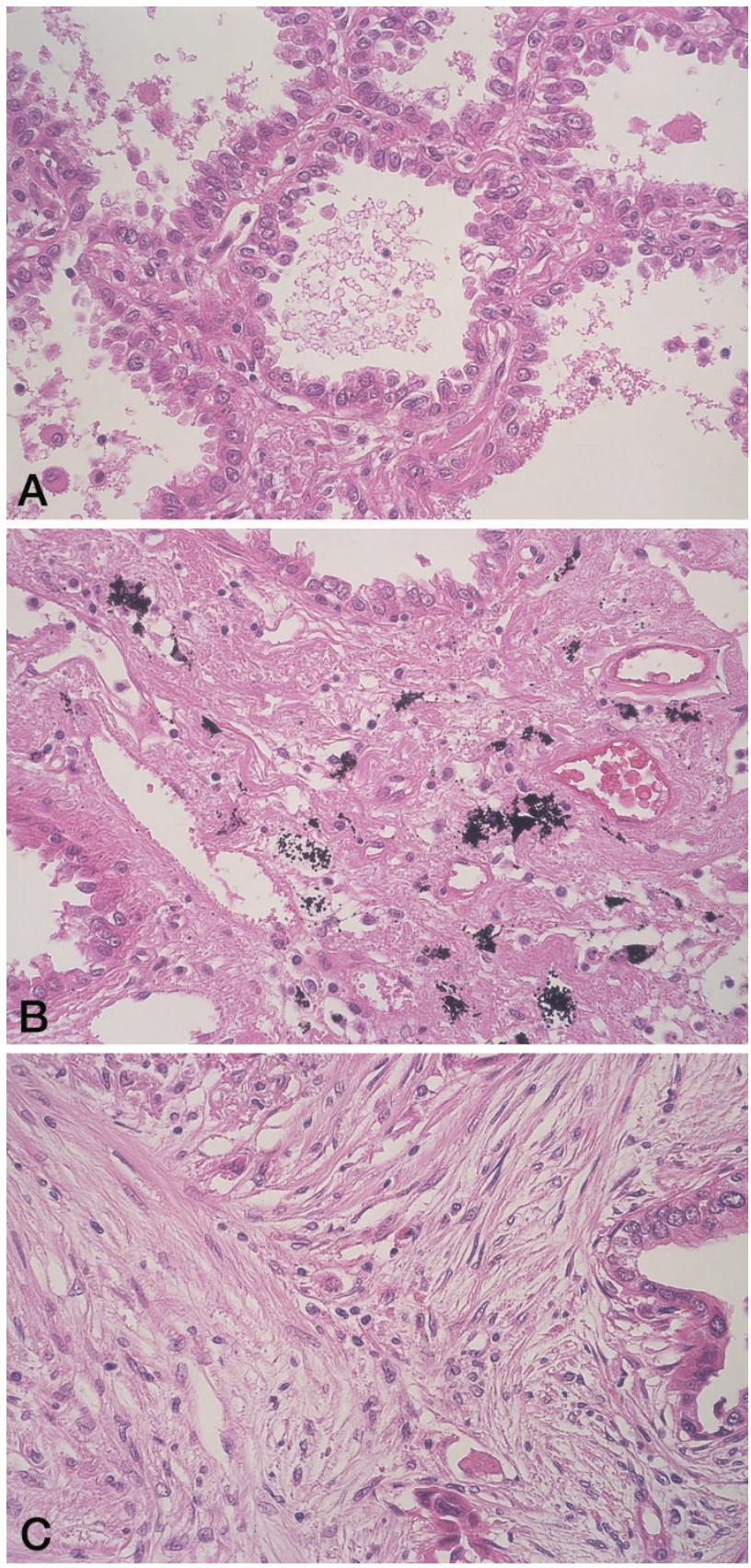

FIGURE 2. Panels indicating the three areas evaluated in this study. A, lepidic growth region. Tumor cells show the replacementgrowth pattern on the wall of preexisting alveoli. B, collapsed alveolar region. Formation of fibrotic foci caused by collapse of the alveoli, where elastic fibers are markedly increased and the alveolar framework is collapsed but not destroyed, is seen. C, scarred region. A fibrotic focus (proliferation of collagen fibers) caused by destruction of existing bronchioloalveolar structure is seen in the central zone of the tumor.

\section{Statistical Analysis}

Univariate analyses of the correlation between histological type and BM preservation and between histological characteristics and lymph node metastasis were performed by the $\chi^{2}$ test. The survival rates were calculated by the Kaplan-Meier method. Differences were considered to be statistically significant when the probability value was less than .05 . 


\section{RESULTS}

\section{Histological Typing}

First, the 72 replacement-growth-type tumors selected were grouped into 4 histological subtypes based on two histological features: preservation of the preexisting elastic framework as described by Eto et al. (20) and the presence of fibrotic foci according to whether there was proliferation of elastic and collagen fiber as described by Clayton (21). In this grouping, 2 cases with no fibrotic foci had a well-preserved preexisting elastic framework. There was no tumor, that had two pathological features together, such as no fibrotic foci and elastic framework destruction. Seventy cases had fibrotic foci in the tumors, including 17 with elastic framework preservation and 53 with elastic framework destruction. On the basis of these findings, we grouped these tumors into the following three distinctive histological structural patterns (Table 2).

\section{Type I: bronchioloalveolar carcinoma without fibrotic foci}

This type of tumor shows the replacementgrowth type of alveolar lining cells (Fig. 3A). Existing bronchioloalveolar structures and elastic framework are well preserved. No elastic fiber condensation or collagen fiber synthesis can be detected in any region of the tumor. It is distinguished from atypical adenomatous hyperplasia by its increased cellularity and cellular atypia.

\section{Type II: sclerosing bronchioloalveolar carcinoma without elastic framework destruction}

This type of tumor also partially shows replacement growth of alveolar lining cells resembling Type I, but fibrotic foci caused by the collapse of preexisting bronchioloalveolar structures are observed in these tumors (Fig. 3B). Condensation of elastic fibers is observed in the fibrotic foci, but the elastic framework is not destroyed. No proliferation of collagen fibers is detected. The alveolar septa in this collapsed region are thickened.

\section{Type III: sclerosing bronchioloalveolar carcinoma with elastic framework destruction}

This type of tumor also partially shows replacement growth of alveolar lining cells resembling

TABLE 2. Histological Typing of the ReplacementGrowth Type of Adenocarcinoma

\begin{tabular}{lcccc}
\hline & \multicolumn{3}{c}{ Histological Features } & \\
\cline { 2 - 4 } Type & $\begin{array}{c}\text { Replacement } \\
\text { Growth }\end{array}$ & $\begin{array}{c}\text { Fibrotic } \\
\text { Foci }\end{array}$ & $\begin{array}{c}\text { Elastic } \\
\text { Framework } \\
\text { Destruction }\end{array}$ & \\
\hline I & + & - & - & 2 \\
II & + & + & - & 17 \\
III & + & + & + & 53 \\
\hline
\end{tabular}

Type I and II. However, fibrotic foci caused by destruction of preexisting bronchioloalveolar structures are observed in the tumors. Collagen fiber synthesis is observed in the fibrotic foci, and the elastic framework is severely distorted or none is detected (Fig. 3C). Collagen fiber synthesis is suggested by foci of active fibroblastic proliferation. In these foci, large nuclei of the proliferation fibroblasts and endothelial cells of small vessels are prominent, and often accompanied by collagenization. Small solid nests of invasive cancer cells are sometimes observed among the collagen fibers.

When the WHO histological classification of adenocarcinoma of the lung was applied to our three histological types, Type I and II tumors were bronchioloalveolar carcinomas, and Type III was adenocarcinoma mixed bronchioloalveolar subtype (19).

Each tumor was histologically subtyped by two pathologists (TY, TK) after a review of slides stained with HE and Victoria-blue for elastic fiber detection. Two tumors were Type I, 17 were Type II, and 53 were Type III (Table 2).

\section{Immunohistochemical Analysis}

Table 3 shows BM preservation in the LG region. Because there were only two Type I tumors, BM preservation was statistically analyzed in Type II and III. The three BM components were well preserved in both types of tumors (Fig. 4). The differences in BM preservation between tumor types were not statistically significant in regard to any of the BM components. Table 4 shows BM preservation in the collapsed alveolar region. BM preservation was significantly lost in Type III tumors (Type IV, $P=.02$; $7 \mathrm{~S}, P=.02$; laminin, $P=.01$; Fig. 5). In the scarred region, there was an abundant amount of collagen fibers, and no BM could be detected by immunohistochemical staining (Table 5; Fig. 6). We concluded that the BM had been largely destroyed by tumor cell invasion and that collagenization had occurred in the scarred region.

We also examined the correlation between histological characteristics, such as histological type, degree of BM destruction, lymph vessel invasion, vascular invasion, and lymph node metastasis (Table 6). When even one BM component was completely destroyed in any region of the tumor, it was considered to be a tumor with BM destruction. Eleven of the 72 cases had pathologically confirmed lymph node metastasis. All were Type III and tumors with BM destruction. There were significant correlations between all histological characteristics and lymph node metastasis.

Figure 7 shows the survival curves by histological type. The 5-year survival rate of the patients with 

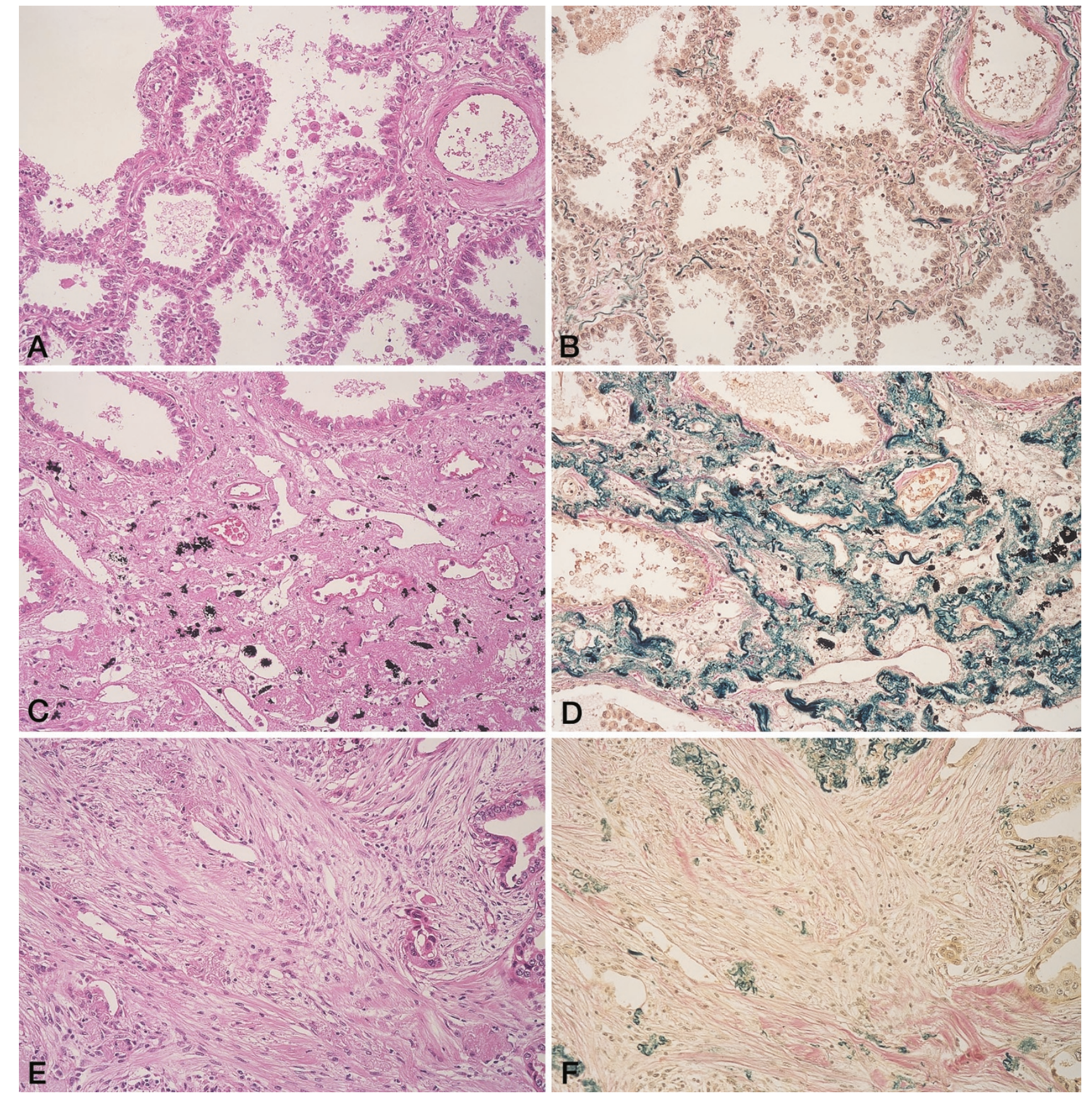

FIGURE 3. Histological types of replacement-growth-type adenocarcinomas of the lung. Top, Type I tumor. All tumor cells show the replacement growth pattern and no fibrotic foci can be seen (A). Existing elastic framework are well preserved (B). Middle, Type II tumor. Fibrotic foci due to collapse of the alveolar structure are evident in the tumor (C). Condensation of elastic fibers is observed in the fibrotic foci (D). Bottom, Type III tumor. Fibrotic foci caused by destruction of existing bronchioloalveolar structures (proliferation of collagen fibers) are evident in the tumor (E). The elastic framework is severely distorted and cannot be detected $(\mathbf{F})$.

Type I and II tumors was $100 \%$, whereas it was 92.5\% in those with Type III tumors. Because none of the patients with Type I and II tumors died and only 4 patients with Type III tumors died of tumors, statistical analysis according to differences in histological types were not performed. In addition, Figure 8 shows that the 5-year survival rate of the patients with BM-preserved tumors was $100 \%$, versus $92.5 \%$ in those with BM-destroyed tumors.

\section{DISCUSSION}

Shimosato et al. (6) reported that the characteristics of the central fibrotic focus are probably more important than tumor size for predicting the outcome of patients with peripheral adenocarcinomas less than $3 \mathrm{~cm}$ in diameter. Kurokawa et al. (22) confirmed that grade of fibrotic scarring (central fibrosis) was one of the most important prognostic 
TABLE 3. BM Preservation in Lepidic Growth Regions

\begin{tabular}{|c|c|c|c|c|c|c|c|c|c|}
\hline \multirow{2}{*}{ BM Preservation } & \multicolumn{3}{|c|}{ Type IV Collagen* } & \multicolumn{3}{|c|}{ 7S Collagen** } & \multicolumn{3}{|c|}{$\operatorname{Laminin}^{* * *}$} \\
\hline & I & II & III & I & II & III & I & II & III \\
\hline $\mathrm{CP}$ & 2 & 15 & 43 & 2 & 16 & 50 & 2 & 16 & 41 \\
\hline $\mathrm{PD}$ & 0 & 2 & 6 & 0 & 0 & 2 & 0 & 0 & 5 \\
\hline $\mathrm{CD}$ & 0 & 0 & 4 & 0 & 1 & 1 & 0 & 1 & 7 \\
\hline
\end{tabular}

II vs. III: ${ }^{*} P=0.51,{ }^{* *} P=0.51,{ }^{* * *} P=0.27$.

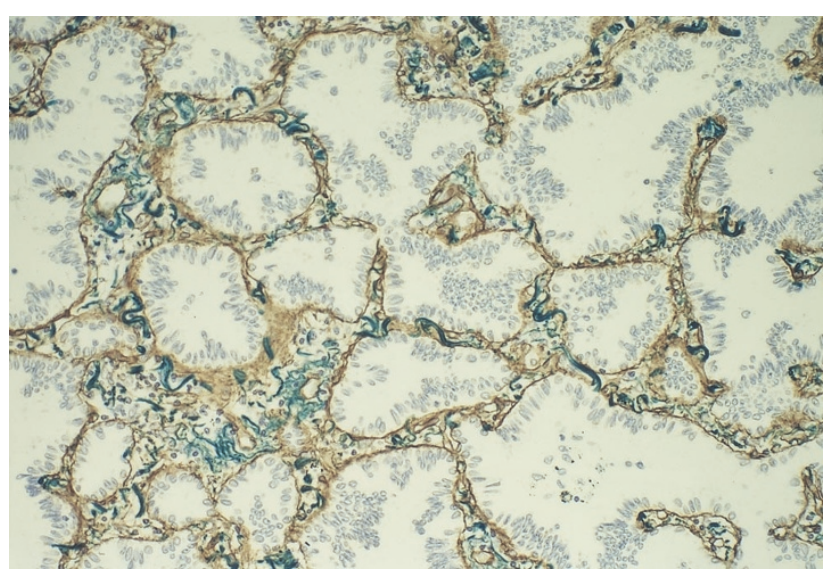

FIGURE 4. Immunohistochemistry of BM components. The BM in this lepidic growth region is well preserved (Type IV collagen).

factors for small bronchioloalveolar carcinoma and that it was often absent in small cancers cured by surgery. In addition, Noguchi et al. (7) reported finding that small LBACs, under $2 \mathrm{~cm}$, with active fibroblastic proliferation had a poor prognosis. These findings indicated that early invasion had already started in small adenocarcinomas with central fibrotic scars.

In this study, the LG region was found at the periphery of the tumors, where the three BM components were well preserved. We therefore concluded that invasive growth probably did not start in this area, the same as in a previous study (23). BM preservation was significantly lost in the collapsed alveolar region of Type III tumors, which showed significant lymph node metastasis and a poor outcome. Although the collapsed alveolar pattern of Type III tumors is very similar to that of Type II tumors, tumor invasion must have already started in this region of Type III tumors. There was an abundant amount of collagen fibers in the scarred region, and the BM could not be detected by immunohistochemical staining. We concluded

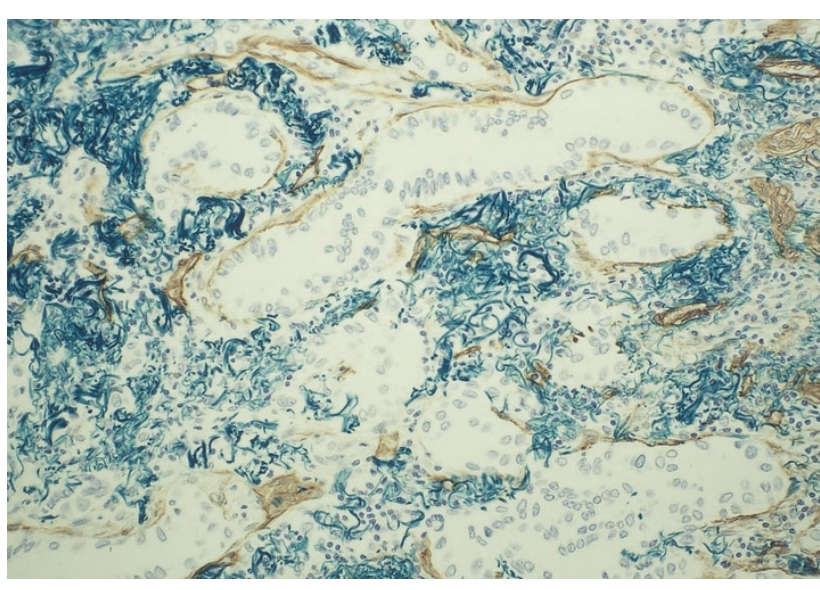

FIGURE 5. BM preservation in the area of collapsed alveoli of Type III tumors. The BM is extensively disrupted (Type IV collagen).

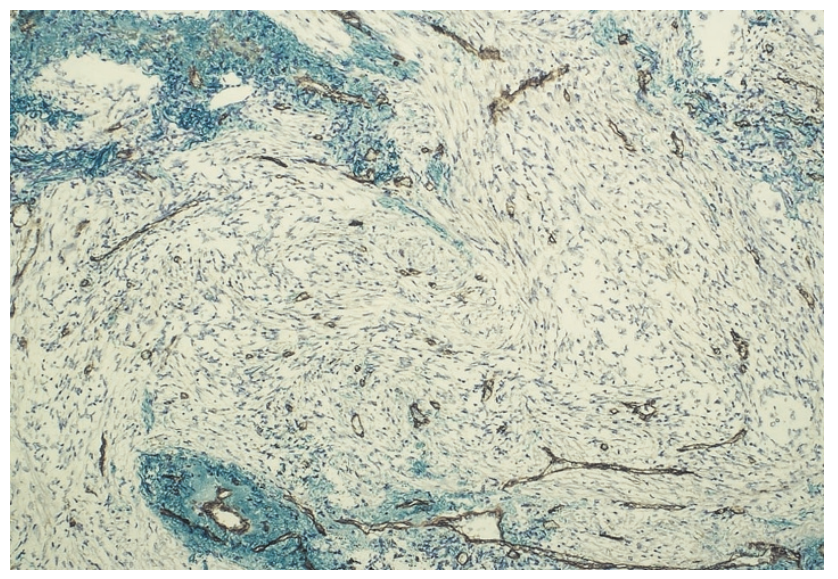

FIGURE 6. BM preservation in the area of the scarred region of a Type III tumor. No BM is detected (laminin).

that the BM had been largely destroyed by tumor cell invasion and that collagenization had occurred in this region. Thus, lymph node metastasis was significantly greater in the Type III tumors.

In 1999, Travis et al. (19) published the WHO histological classification of tumors of the lung, in

TABLE 4. BM Preservation in Collapsed Alveolar Regions

\begin{tabular}{|c|c|c|c|c|c|c|}
\hline \multirow{2}{*}{ BM Preservation } & \multicolumn{2}{|c|}{ Type IV Collagen* } & \multicolumn{2}{|c|}{ 7S Collagen** } & \multicolumn{2}{|c|}{ Laminin $^{* * *}$} \\
\hline & $\overline{\mathrm{II}}$ & $\overline{\text { III }}$ & II & $\overline{\text { III }}$ & $\overline{\mathrm{II}}$ & $\overline{\text { III }}$ \\
\hline CP & 11 & 14 & 13 & 21 & 7 & 6 \\
\hline PD & 3 & 15 & 2 & 7 & 3 & 7 \\
\hline $\mathrm{CD}$ & 3 & 24 & 2 & 25 & 7 & 40 \\
\hline
\end{tabular}

II vs. III: ${ }^{*} P=0.02,{ }^{* *} P=0.02,{ }^{* *} P=0.01$. 
TABLE 5. BM Preservation in Scarred Region

\begin{tabular}{|c|c|c|c|}
\hline BM Preservation & $\begin{array}{l}\text { Type IV } \\
\text { Collagen }\end{array}$ & $\begin{array}{c}7 \mathrm{~S} \\
\text { Collagen }\end{array}$ & Laminin \\
\hline & III & III & III \\
\hline $\mathrm{CP}$ & 0 & 0 & 0 \\
\hline $\mathrm{PD}$ & 0 & 0 & 0 \\
\hline $\mathrm{CD}$ & 53 & 53 & 53 \\
\hline
\end{tabular}

TABLE 6. Lymph Node (LN) Metastasis According to Histological Characteristics

\begin{tabular}{lrrr}
\hline \multirow{2}{*}{ Parameter } & \multicolumn{2}{c}{ LN } & \\
& \multicolumn{2}{c}{ Metastasis } & P Value \\
\cline { 2 - 3 } & - & + & \\
\hline Histological type & 19 & 0 & .03 \\
$\quad$ I and II & 42 & 11 & \\
III & 18 & 0 & .05 \\
BM destruction & 43 & 11 & \\
- & & & \\
+ & 55 & 3 & $<.01$ \\
Lymph vessel invasion & 6 & 8 & \\
- & & & \\
+ & 55 & 6 & \\
Vascular invasion & 6 & 5 & \\
- & & & \\
+ & & & \\
\hline
\end{tabular}

which they subclassified adenocarcinoma of the lung into acinar, papillary, bronchioloalveolar carcinoma, solid adenocarcinoma with mucin, adenocarcinoma with mixed subtypes and variants. In particular, bronchioloalveolar carcinoma has been defined as an adenocarcinoma with a pure bronchioloalveolar growth pattern and no evidence of stromal vascular or pleural invasion. If an invasive component is identified, then the tumor is classified as adenocarcinoma, mixed bronchioloalveolar subtype, rather than pure bronchioloalveolar carcinoma (19). In our series of adenocarcinomas, the BM component was very well preserved in Type I and II tumors. On the other hand, BM preservation was significantly lost in Type III tumors. Therefore, when the WHO pathological criteria for adenocarcinoma of the lung was applied to our series of replacement-growth type of small peripheral adenocarcinomas, Type I and II tumors were bronchioloalveolar carcinomas, and Type III was adenocarcinoma mixed bronchioloalveolar subtype. In fact, the patients with Type I and II tumors had a good prognosis.

In this study, we could not distinguish between the original $\mathrm{BM}$ of the alveoli and newly formed $\mathrm{BM}$ produced by tumor cells. We were also unable to distinguish between discontinuous BM caused by cancer invasion and caused by mechanical destruction from alveolar collapse. It may become possible to use degree of BM preservation as a factor significantly correlated with invasion and metastasis if it becomes possible to distinguish different BM types by other biological markers.

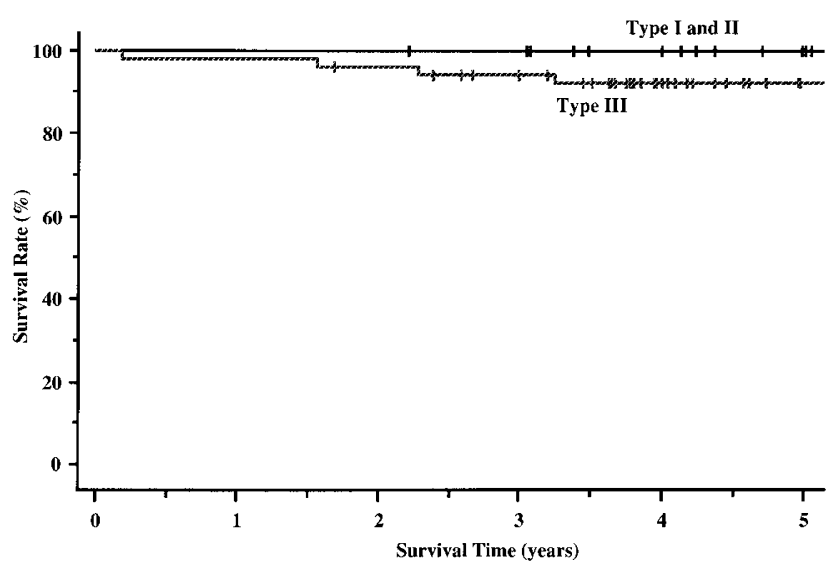

FIGURE 7. Survival curve of patients with Type I and II tumors versus patients with Type III tumors.

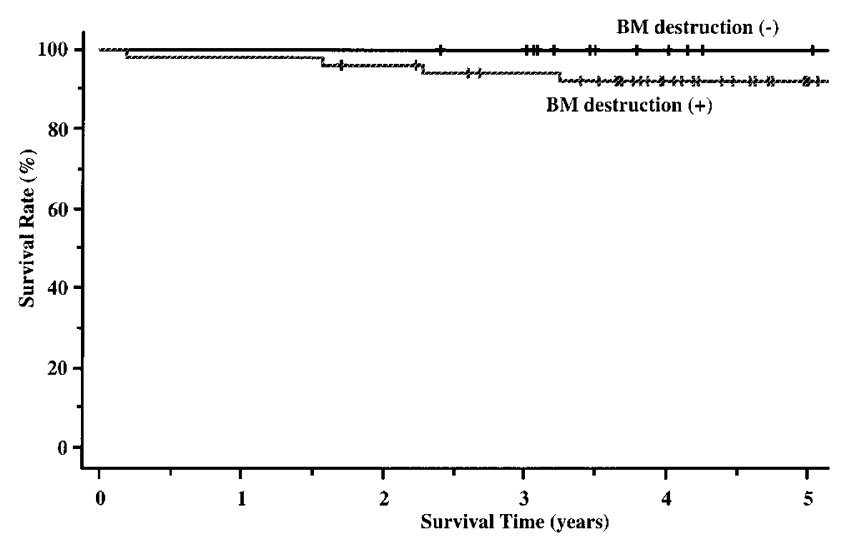

FIGURE 8. Survival curve of patients with BM-preserved and BMdestroyed tumors.

Other histochemical stains such as periodic acidSchiff and reticulin also reveal positive staining for $\mathrm{BM}$. Therefore we tried to evaluate the degree of BM preservation in our series using periodic acidSchiff stain. However, because it demonstrated only outlines of BM, it was difficult to evaluate the degree of BM preservation (data not shown). Additionally, although BM are largely composed of Type IV collagen and laminin, reticulin stains only demonstrate reticular fibers, which consist of very thin fibers of mainly Type III collagen (24). Consequently, it is not possible to evaluate BM preservation precisely by reticulin stain. To evaluate the degree of BM preservation, immunohistochemical staining for Type IV collagen and laminin are needed.

No lymph node metastasis was detected in Type I or II tumors, and the 5-year survival rate was $100 \%$. We think that these tumor types are noninvasive adenocarcinoma because the BM component was very well preserved. It may be possible to perform partial resection of Type I and II tumors clinically. A clinical trial of minimal dissection of Type I and II tumors is presently in progress at our 
institute. According to the protocol of this trial, we are examining frozen sections of peripheral adenocarcinomas less than $2 \mathrm{~cm}$ that had been rapidly stained for elastic fibers to detect alveolar framework destruction. If the pathologists diagnose the tumor as Type I or II, partial resection is performed. The results of this trial will be reported in the future.

Type IV collagenase, matrix metalloproteinase (MMP) 2, and MMP 9, which are secreted by cancer cells, have been shown to play an important role in the degradation of BM (25-29). Expression of these enzymes by cancer cells has been found to be correlated with the malignant potential of various cancers (30-36). Expression of these enzymes may be responsible for tumor invasion and metastasis of $\mathrm{T} 1$ adenocarcinoma of the lung (37). We are currently investigating correlations between expression of these enzymes and BM preservation in the same specimens. We suspect that Type I tumors express inactive MMPs and that when they advance to Type II, the tumors begin to activate the enzymes, and they invade the extracellular matrix. Cancer invasion and collagenization must form the scarred regions observed in Type III tumors.

In conclusion, we evaluated BM destruction in three different histological areas of the same tumor and correlated it with metastasis. Our findings suggested that Type I and II tumors are noninvasive and that Type III tumors are invasive. The pattern of BM destruction observed in the collapsed alveolar area of Type III tumors must be the first step in tumor invasion. Moreover, the three types classified on the basis of BM preservation serve as a good indicator for limited surgery of peripheral-replacementtype adenocarcinoma of the lung.

Acknowledgments: We thank Drs. Shoichiro Tsugane and Satoshi Sasaki (National Cancer Center Research Institute East) for their assistance in statistical analysis.

\section{REFERENCES}

1. Martini N, Beattie EJ Jr. Results of surgical treatment in Stage I lung cancer. J Thorac Cardiovasc Surg 1977;74:499-505.

2. Thomas PA Jr, Rubinstein L. Malignant disease appearing late after operation for T1 N0 non-small-cell lung cancer. The Lung Cancer Study Group. J Thorac Cardiovasc Surg 1993;106:1053-8.

3. Nesbitt JC, Putnam JB Jr, Walsh GL, Roth JA, Mountain CF. Survival in early-stage non-small cell lung cancer. Ann Thorac Surg 1995;60:466-72.

4. Naruke T, Goya T, Tsuchiya R, Suemasu K. Prognosis and survival in resected lung carcinoma based on the new international staging system. J Thorac Cardiovasc Surg 1988;96: $440-7$.

5. Williams DE, Pairolero PC, Davis CS, Bernatz PE, Payne WS, Taylor WF, et al. Survival of patients surgically treated for stage I lung cancer. J Thorac Cardiovasc Surg 1981;82:70-6.
6. Shimosato Y, Suzuki A, Hashimoto T, Nishiwaki Y, Kodama $\mathrm{T}$, Yoneyama $\mathrm{T}$, et al. Prognostic implications of fibrotic focus (scar) in small peripheral lung cancers. Am J Surg Pathol 1980;4:365-73.

7. Noguchi M, Morikawa A, Kawasaki M, Matsuno Y, Yamada T, Hirohashi S, et al. Small adenocarcinoma of the lung. Histologic characteristics and prognosis. Cancer 1995;75:284452.

8. Yamashiro K, Yasuda S, Nagase A, Hirata T, Nojima T, Nagashima K. Prognostic significance of an interface pattern of central fibrosis and tumor cells in peripheral adenocarcinoma of the lung. Hum Pathol 1995;26:67-73.

9. Liotta LA. Tumor invasion and metastases: role of the basement membrane. Warner- Lambert Parke-Davis Award lecture. Am J Pathol 1984;117:339-48.

10. Liotta LA, Stetler-Stevenson WG. Tumor invasion and metastasis: an imbalance of positive and negative regulation. Cancer Res 1991;51:5054s-9s.

11. Liotta LA, Steeg PS, Stetler-Stevenson WG. Cancer metastasis and angiogenesis: an imbalance of positive and negative regulation. Cell 1991;64:327-36.

12. Terranova VP, Hujanen ES, Martin GR. Basement membrane and the invasive activity of metastatic tumor cells. J Natl Cancer Inst 1986;77:311-6.

13. Nakagawa H, Yagihashi S. Expression of type IV collagen and its degrading enzymes in squamous cell carcinoma of lung. Jpn J Cancer Res 1994;85:934-8.

14. Havenith MG, Arends JW, Simon R, Volovics A, Wiggers T, Bosman FT. Type IV collagen immunoreactivity in colorectal cancer. Prognostic value of basement membrane deposition. Cancer 1988;62:2207-11.

15. Albrechtsen R, Nielsen M, Wewer U, Engvall E, Ruoslahti E. Basement membrane changes in breast cancer detected by immunohistochemical staining for laminin. Cancer Res 1981;41:5076-81.

16. ten Velde GP, Havenith MG, Volovics A, Bosman FT. Prognostic significance of basement membrane deposition in operable squamous cell carcinomas of the lung. Cancer 1991;67:3001-5.

17. Mori M, Kohli A, Baker SP, Savas L, Fraire AE. Laminin and cathepsin B as prognostic factors in stage I non-small cell lung cancer: are they useful? Mod Pathol 1997;10:572-7.

18. Yurchenco PD, Schittny JC. Molecular architecture of basement membranes. FASEB J 1990;4:1577-90.

19. Travis WD, Colby TV, Corrin B, Shimosato Y, Brambilla E. Histological typing of lung and pleural tumours. 3rd ed. Berlin: Springer; 1999.

20. Eto T, Suzuki H, Honda A, Nagashima Y. The changes of the stromal elastotic framework in the growth of peripheral lung adenocarcinomas. Cancer 1996;77:646-56.

21. Clayton F. Bronchioloalveolar carcinomas. Cell types, patterns of growth, and prognostic correlates. Cancer 1986;57: 1555-64.

22. Kurokawa T, Matsuno Y, Noguchi M, Mizuno S, Shimosato Y. Surgically curable "early" adenocarcinoma in the periphery of the lung. Am J Surg Pathol 1994;18:431-8.

23. Paakko P, Risteli J, Risteli L, Autio-Harmainen H. Immunohistochemical evidence that lung carcinomas grow on alveolar basement membranes. Am J Surg Pathol 1990;14:46473.

24. Rosai J. Ackerman's surgical pathology. 8th ed. St. Louis: Mosby; 1996.

25. Canete-Soler R, Litzky L, Lubensky I, Muschel RJ. Localization of the $92 \mathrm{kd}$ gelatinase mRNA in squamous cell and adenocarcinomas of the lung using in situ hybridization. Am J Pathol 1994;144:518-27.

26. Sato H, Takino T, Okada Y, Cao J, Shinagawa A, Yamamoto E, Seiki M. A matrix metalloproteinase expressed on the surface 
of invasive tumour cells [see comments]. Nature 1994;370: 61-5.

27. Levy AT, Cioce V, Sobel ME, Garbisa S, Grigioni WF, Liotta $\mathrm{LA}$, et al. Increased expression of the Mr 72,000 type IV collagenase in human colonic adenocarcinoma. Cancer Res 1991;51:439-44.

28. Boag AH, Young ID. Increased expression of the 72-kd type IV collagenase in prostatic adenocarcinoma. Demonstration by immunohistochemistry and in situ hybridization. Am J Pathol 1994;144:585-91.

29. Davies B, Miles DW, Happerfield LC, Naylor MS, Bobrow LG, Rubens RD, et al. Activity of type IV collagenases in benign and malignant breast disease. Br J Cancer 1993;67: 1126-31.

30. Kawahara E, Okada Y, Nakanishi I, Iwata K, Kojima S, Kumagai S, et al. The expression of invasive behavior of differentiated squamous carcinoma cell line evaluated by an in vitro invasion model. Jpn J Cancer Res 1993;84:409-18.

31. Brown PD, Bloxidge RE, Stuart NS, Gatter KC, Carmichael J. Association between expression of activated 72-kilodalton gelatinase and tumor spread in non-small-cell lung carcinoma. J Natl Cancer Inst 1993;85:574-8.

32. Nakajima M, Morikawa K, Fabra A, Bucana CD, Fidler IJ. Influence of organ environment on extracellular matrix degra- dative activity and metastasis of human colon carcinoma cells [see comments]. J Natl Cancer Inst 1990;82:1890-8.

33. Garbisa S, Scagliotti G, Masiero L, Di Francesco C, Caenazzo C, Onisto M, et al. Correlation of serum metalloproteinase levels with lung cancer metastasis and response to therapy. Cancer Res 1992;52:4548-9.

34. Davies B, Waxman J, Wasan H, Abel P, Williams G, Krausz T, et al. Levels of matrix metalloproteases in bladder cancer correlate with tumor grade and invasion. Cancer Res 1993; 53:5365-9.

35. Grigioni WF, D’Errico A, Fiorentino M, Baccarini P, Onisto M, Caenazzo C, et al. Gelatinase A (MMP-2) and its mRNA detected in both neoplastic and stromal cells of tumors with different invasive and metastatic properties. Diagn Mol Pathol 1994;3:163-9.

36. Azzam HS, Arand G, Lippman ME, Thompson EW. Association of MMP-2 activation potential with metastatic progression in human breast cancer cell lines independent of MMP-2 production. J Natl Cancer Inst 1993;85:1758-64.

37. Kodate M, Kasai T, Hashimoto H, Yasumoto K, Iwata Y, Manabe H. Expression of matrix metalloproteinase (gelatinase) in T1 adenocarcinoma of the lung. Pathol Int 1997;47: 461-9. 